\title{
O ser mulher e sua alteridade no âmbito da saúde biopsicossocial na pós-contemporaneidade: uma revisão narrativa
}

\author{
Being a woman and her otherness in the context of biopsychosocial health in post- \\ contemporaneity: a narrative review
}

\begin{abstract}
Ser mujer y su alteridad en el contexto de la salud biopsicosocial en la postcontemporaneidad: una revisión narrativa
\end{abstract}

lasminny Loiola Teixeira ${ }^{1 *}$, Aline Sampaio Rolim de Sena ${ }^{1}$, Giovanna Sales de Oliveira ${ }^{1}$, Brunna Nayara Alves Sousa Rolim de Sena ${ }^{1}$, Yuri Monteiro Bezerra ${ }^{2}$, Tayne Sales Silva ${ }^{1}$, Woneska Rodrigues Pinheiro ${ }^{1}$, Rachel de Sá Barreto Luna Callou Cruz ${ }^{1}$.

\section{RESUMO}

Objetivo: Descrever e revisar sobre a saúde da mulher no contexto biopsicossocial e os impactos provenientes da sua alteridade perpassando o contexto histórico e cultural até a pós-contemporaneidade. Revisão bibliográfica: Apresentou-se as vivências da mulher na pós-contemporaneidade compreendendo a multiplicidade das suas lutas, considerando sua alteridade e priorizando discussões que fomentem o cuidado dos profissionais de saúde. Levou-se em consideração o rol de aspectos apresentados no tocante a alteridade do ser e pertencer ao engodo do sexo feminino, é importante compreender o conceito de alteridade feminina como sendo a inserção interpessoal da mulher no meio social como indivíduo capaz de resignar sua própria história restituindo seu valor, de forma singular e autônoma, bem como delimitando e vivendo os seus espaço de falar e existir, afirmando, dessa forma, sua feminilidade e rompendo com uma posição secundária em termos de cuidados e de todas as particularidades que respaldam a significância da sua saúde e sobrevivência. Considerações finais: Diante do exposto, percebeu-se que a compreensão do modo como a mulher se apresenta na atualidade, revela a importância da vivência da sua alteridade de forma digna, consciente e libertadora, bem como da atuação ética e da humanização nas práticas em saúde.

Palavras-chave: Mulher, Alteridade, Enfermagem, Biopsicossocial, Psicologia.

\section{ABSTRACT}

Objective: Describe and review women's health in the biopsychosocial context and the impacts arising from their otherness, passing through the historical and cultural context to post-contemporaneity. Bibliographic review: The experiences of women in post-contemporaneity were presented, understanding the multiplicity of their struggles, considering their otherness and prioritizing discussions that encourage the care of health professionals. The list of aspects presented regarding the otherness of being and belonging to the female enticement was taken into account. It is important to understand the concept of female otherness as the interpersonal insertion of women in the social environment as an individual capable of resigning their own history restoring its value, in a singular and autonomous way, as well as delimiting and living its space to speak and exist, thus affirming its femininity and breaking with a secondary position in terms of care and all the particularities that support the significance of your health and survival. Final considerations: Given the above, it was realized that understanding the way women present themselves today reveals the importance of experiencing their otherness in a dignified, conscious and liberating manner, as well as the ethical performance and humanization in practices in health.

Keywords: Woman, Otherness, Nursing, Biopsychosocial, Psychology.

1 Universidade Regional do Cariri (URCA), Crato - CE. `E-mail: iasminnyloiolapsi@gmail.com

2 Centro Universitário Doutor Leão Sampaio (UNILEÃO), Juazeiro do Norte - CE. 


\section{RESUMEN}

Objetivo: Describir y revisar la salud de las mujeres en el contexto biopsicosocial y los impactos derivados de su alteridad, pasando del contexto histórico y cultural a la post-contemporaneidad. Revisión bibliográfica: Se presentaron las vivencias de las mujeres en la post-contemporaneidad, entendiendo la multiplicidad de sus luchas, considerando su alteridad y priorizando discusiones que incentiven el cuidado de los profesionales de la salud. Se tomó en cuenta el listado de aspectos presentados sobre la alteridad del ser y la pertenencia a la seducción femenina. Es importante entender el concepto de alteridad femenina como la inserción interpersonal de la mujer en el ámbito social como individuo capaz de renunciar a su propia historia. restituir su valor, de manera singular y autónoma, así como delimitar y vivir su espacio para hablar y existir, afirmando así su feminidad y rompiendo con una posición secundaria en cuanto al cuidado y todas las particularidades que sustentan la significación de su salud y supervivencia. Consideraciones finales: Teniendo en cuenta lo anterior, se percató que comprender la forma en que las mujeres se presentan hoy revela la importancia de vivir su alteridad de manera digna, consciente y liberadora, así como el desempeño ético y la humanización en las prácticas en salud.

Palabras clave: Mujeres, Alteridad, Enfermería, Biopsicosocial, Psicología.

\section{INTRODUÇÃO}

Ao longo dos anos, foram observados muitos vieses socioeconômicos e culturais, construídos por uma sociedade que culturalmente diferencia o feminino do masculino, no que diz respeito ao espaço de dominação e hierarquia, sendo a mulher o sujeito sem evidência e prestígio no âmbito social, por diversas vezes, vítima de comportamentos machistas e preconceituosos (SANTOS LG, 2019).

Apesar das discussões, lutas e construções acerca do gênero feminino, ainda se revela uma realidade do "Ser Mulher" ausente de direitos, mesmo com avanços constitucionais, socioculturais e do papel protagonista em diversas instâncias da sociedade, configurando assim, um quadro de alteridade feminina inferior à concepção culturalmente machista, configurada a respeito do ser mulher (BEAUVOIR S, 1967).

O termo "ser mulher" é um tanto quanto expansivo. Porém, o que ocorre é que, nos diversos discursos científicos e/ou populares, esta expressão passa a ser limitada somente ao sexo, reduzindo-se ao campo biológico, todavia pode ser concebido como uma construção social e cultural da identidade de gênero (BUTLER J, 2003).

Beauvoir S (1967) endossa que "ninguém nasce mulher, torna-se mulher", ratificando a experiência que pode ser muito bem traduzida diante da expressão "ser mulher", uma vez que compreender o fenômeno existencial de ser uma mulher, está para além de uma simples definição de natureza biológica, trata-se de uma fusão de concepções, atitudes e sentimentos.

Esse fenômeno cultural de reduzir a mulher ao sexo feminino, ao conceito biológico, desconsidera muitos elementos significantes da sua existência em sociedade, como o seu próprio cotidiano, os seus sentimentos e o que produz a partir das suas concepções e desejo. Em seu estudo, Martino LMS e Marques ACS (2019) esclarecem que o outro é percebido e compreendido enquanto alteridade, quando este passa a ser absolutamente aquilo que dita a sua vivência com o social, com o outro, sendo a representação das suas experiências o que se constitui a partir das relações vivenciadas com o outro.

Assim, falar sobre alteridade feminina, amplia a reflexão sobre tal temática e evidencia a presença da mulher em um meio de multirrelações, sendo fundamental para a compreensão de que o ideal de homem tem modificado o espaço da mulher na pós-contemporaneidade (CHACON DRA, 2015).

Dessa forma, a alteridade se manifesta no dia a dia da vivência do mundo feminino sem a presunção de detalhar o modo do viver enquanto identidade, não tendo relação de oposição com o mundo masculino, muito menos tentando reforçar as inúmeras diferenças entre os sexos, mas sim a partir da ótica do espaço de produção desse sujeito, da forma como a mulher se representa no mundo e frente ao Outro, restaurando, assim, o seu valor enquanto ser que não precisa submeter-se ao ideal masculino (BEAUVOIR S, 1967). 
Nessa perspectiva, a mulher não pertence apenas a um escopo de conquistas sociais, culturais, mas também é peça fundamental de representações "corporificadas", tendo o seu corpo apenas, em sua maioria, uma função produtiva cuja maternidade é seu principal atributo, visto que ela está inserida em um contexto biopsicossocial e cultural machista. Fatores estes, preponderantes na determinação das condições de saúde-doença (BENHABIB SDC, 1987).

A mulher adoece mais que o homem, mesmo buscando muito mais os serviços de saúde e obtendo longevidade. Dessa forma, após observância dos fatores de risco relacionado a esse público, foi desenvolvido em 2004 a Política Nacional de Atenção Integral à Saúde da Mulher (PNAISM), como estratégia de inclusão e integração no âmbito da atenção à saúde, ampliando os cuidados à mulher, inserindo-a na Rede de Atenção à Saúde (BRETON DL, 2017).

No entanto, mesmo com acesso aos serviços de promoção e prevenção, a Organização Mundial de Saúde (OMS) alerta sobre as doenças de maior incidência na saúde da mulher até o ano de 2019, cujo impacto se apresenta a nível nacional e mundial, com destaque para os transtornos psíquicos, onde $20 \%$ das mulheres tiveram ou terão depressão (CONSELHO NACIONAL DE SECRETARIAS MUNICIPAIS DE SAÚDE, 2019).

O Instituto Nacional de Câncer (INCA) calculou a proporção dos dez tipos de câncer incidentes em 2020 no sexo feminino, com destaque para o câncer de mama com $29 \%, 7$, Cólon e Reto $9,2 \%$, Colo Uterino $7,5 \%$. Com isso, é primordial uma atuação transversal, multiprofissional e transdisciplinar na prevenção e promoção do cuidar, o que deve acontecer primordialmente na Atenção Primária à Saúde (INSTITUO NACIONAL DE CANCÊR, 2020).

Tal realidade é resultante das diversas fragilidades existentes no âmbito do cuidar, em todos os níveis de atenção à saúde, mas também está envolvida por tabus socioculturais na busca preventiva e promocional por esse público. Ações estas, desenvolvidas na Estratégia de Saúde da Família (ESF), local onde a mulher possui maior adesão e comunicação, onde o profissional de enfermagem ocupa espaço de gestão, assistência e liderança, e o psicólogo acolhe de forma multiprofissional em conjuntura com a enfermagem (MOROSSI MVGV, et al., 2018).

Portanto, o presente estudo tem como objetivo descrever a saúde da mulher no contexto biopsicossocial e os impactos provenientes da sua alteridade perpassando o contexto histórico e cultural até a póscontemporaneidade.

\section{REVISÃO BIBLIOGRÁFICA}

As informações encontradas sobre o ser mulher e o modo como ela vivência a pós-contemporaneidade foram apresentadas em três categorias, a saber: Política Nacional de Atenção Integral à Saúde da Mulher no âmbito do Sistema Único de Saúde; A saúde da mulher e os impactos provenientes da alteridade desse ser; Ser mulher na sociedade pós-contemporânea e a atuação do profissional de saúde frente à saúde da mulher.

Todas as categorias abordam a vivência do ser mulher na pós-contemporaneidade, focando na multiplicidade das suas lutas por espaço de existência, respeitando a sua alteridade e priorizando discussões que fomentem o cuidado dos profissionais de saúde, em especial o psicólogo e o enfermeiro, para com o corpo e a sexualidade feminina.

\section{Política Nacional de Atenção Integral à Saúde da Mulher no Âmbito do Sistema Único de Saúde}

O Sistema Único de Saúde (SUS), criado a partir da constituição federal de 1988, garante à população acesso gratuito a todos os serviços de saúde, os quais são embasados em princípios como integralidade, universalidade e equidade (INSTITUTO NACIONAL DE CANCÊR, 2020). Uma das formas idealizadas pelo SUS para contemplar todos os seus objetivos foi através da criação das políticas públicas com enfoque em grupos populacionais separados de acordo com os seus determinantes, como por exemplo, a Política Nacional de Atenção Integral à Saúde da Mulher (PNAISM) (MACHADO CV, et al., 2017). 
A história da criação da PAISM iniciou-se há décadas atras, a partir de movimentos femininos que questionavam o fato de a assistência à saúde da mulher ter como foco apenas a gestação e o puerpério. Nesse interim, em 1986 foi criado o Programa de Atenção Integral à Saúde da Mulher, que em 2004 foi transformado em política pública de saúde, mantendo como objetivo a atenção integral e o respeito a singularidade do ser mulher (CHACON DRA, 2015).

Dessa forma, a partir de 2004, iniciou-se uma nova fase da assistência à saúde desse público, onde aconteceu a ampliação dos serviços ofertados, agora incluindo o direito sexual e reprodutivo, respeitando a autonomia feminina sobre o seu corpo e os seus desejos. Além disso, ampliaram-se as ferramentas para o atendimento a esse ser, incluindo consultas de enfermagem para assistência sexual e reprodutiva, através do planejamento familiar, consultas ginecológicas, entre outras (MINISTÉRIO DA SAÚDE, 2004).

De acordo com a rede de atenção do SUS, a Estratégia de Saúde da Família (ESF) é a porta de entrada do usuário do sistema, com capacidade de solucionar cerca de $80 \%$ das demandas populacionais, assim, a maior parte do atendimento e acompanhamento a mulher é realizado pela ESF, sendo este local um dos responsáveis pela implementação da PNAISM, além das maternidades, realizando ações baseadas na promoção da saúde e prevenção de doenças (SALES OP, et al., 2019).

Mesmo diante da ampliação do cuidado, a gestante ainda ocupa papel primordial nos programas de atenção à saúde da mulher, tendo em vista as inúmeras ocorrências de mortalidade materna e infantil por causas evitáveis, o que levou à criação da rede cegonha, em 2011, com o objetivo de garantir a redução desses casos e oferecer uma assistência completa e mais humanizada, voltada para a mulher e sua família. Dessa forma, a rede cegonha está presente em todos os níveis de atenção, ou seja, da ESF até a maternidade, permitindo que o cuidado seja continuo e aconteça em toda a rede de atenção à saúde (MINISTÉRIO DA SAÚDE, 2011a).

Apesar desta política de saúde e das melhorias no cuidado a esse público, as fragilidades da atuação em saúde, principalmente das gestantes, levam a reincidência da mortalidade materna, considerando que ainda há complicações como a ocorrência de diabetes gestacional, hipertensão, pré-eclâmpsia, eclampsias, dentre outros agravos (MINISTÉRIO DA SAÚDE, 2011b).

Outra dificuldade que a mulher vive neste contexto refere-se aos direitos sexuais e reprodutivos. Os serviços de saúde são fragilizados nesse âmbito, pois oferecem e se preocupam apenas em assistenciar o acompanhamento da gestante, negligenciando outros aspectos. Portanto, salienta-se que as ESF's enfatizem o planejamento familiar e de saúde sexual, realizando ações que integrem a mulher como um todo e não somente para a reprodução (MOROSINI MVGC, et al., 2017).

Diante de questões como gravidez, planejamento, sexualidade e enfermidades experienciadas pelo ser mulher, o índice de transtornos mentais é crescente, a maioria não recebe atenção integral, são em parte ignoradas nos seus direitos, sendo levadas a ocupem um espaço de vulnerabilidade social dentro da política de saúde pública destinada a elas (MINISTÉRIO DA SAÚDE, 2011b).

Portanto, fazem-se necessárias ações e fiscalizações que garantam a efetividade desta política. Renovar a assistência, de modo a envolver a comunidade nas ações desenvolvidas pela ESF, no que concerne principalmente na realização das práticas educativas. Ademais, é importante que os gestores da saúde ampliem seus olhares para o cuidado no âmbito biopsicossocial do ser mulher, garantindo para esta um espaço de significância na sociedade (MACEDO JG e ARRAES R, 2013).

\section{A saúde da mulher e os impactos provenientes da alteridade desse ser}

Desde a concepção de uma criança, o gênero é discutido com teor complexo e de desigualdade, tendo como base influências culturais e sociais impostas pelo determinismo biológico entre os sexos. A imagem do ser mulher é alvo de questionamentos desde o início dos séculos, a começar pela visão teológica representada em algumas passagens cristãs, que enalteceram a mulher e seu corpo, como significantes do pecado e apólogos a Deus (FRANCISCO AM, 2013).

Socialmente, o gênero está posto desde antes do nascimento ao reducionismo biológico, determinando a sua existência em apenas um fator do complexo âmbito que constitui alteridade de um ser. Sua capacidade 
de pensar, agir e falar sempre tendeu a ser menosprezada e restringida às responsabilidades administrativas e domésticas de um lar, mantendo uma função social de respeito e obediência sob às afirmações e imposições masculinas (SODRÉ MP, et al., 2017). Já o homem ao que se propunha era o inverso da mulher, considerado visionário como indivíduo de força, líder, um ser pensante e pertinentemente operante, realidade esta que não é distante do que se vive atualmente (MENDES VR, et al., 2015).

$\mathrm{Na}$ contemporaneidade, a mulher ainda luta pela validação da sua alteridade e existência. Pesquisas apresentam altas taxas de desigualdades nas esferas educacionais, econômicas, políticas e de saúde, impactando diretamente na morbimortalidade das mesmas, resultado do que estão mais passíveis de patologias devido aos desgastes físicos e mentais que sofrem (BOTTI NCL, et al., 2013).

Escolas são mais frequentadas por meninos do que por meninas, no trabalho só ganham $77 \%$ do salário, equiparado ao que o sujeito homem com as mesmas atribuições ganharia, evidenciando assim as desigualdades de gênero, o que compromete seu bem-estar biopsicossocial. Além da desvalorização, sofrem violência em vários espaços (COELHO S e PORTO YF, 2013).

Em níveis populacionais, as mulheres representam cerca $51,7 \%$ do Brasil, enquanto homens são compostos por volta de $48,3 \%$. Essa realidade se repete em termos de longevidade de vida, as mulheres vivem mais que os homens, sendo 79,9 anos para 72,7 anos. Esses números eram bem decrescentes em anos atrás, em 1940 a expectativa de vida era apenas de 45 anos, principalmente quando se tratava das mulheres. Dados estes que estão relacionados com a qualidade de vida que cada sujeito escolhe para si, o que evidencia ainda mais a luta da mulher por espaço e cuidado (FRANCISCO AM, 2013).

Vale salientar que a maior parte dos agravos e problemas de saúde que acometem a mulher são provenientes, principalmente, dos contextos sociais, que ainda englobam a categoria feminina como seres à margem da virilidade de ser produtivo, pela discriminação, sobrecarga de responsabilidades, raça, etnia, gênero e classe social. As mulheres vivem mais do que os homens, entretanto adoecem com maior frequência (BRETON DL, 2017).

Em meio a tantas lacunas, o público feminino sempre possuiu uma maior adesão ao Sistema Único de Saúde (SUS), seja por uso próprio ou para acompanhamento de algum familiar. Em décadas atrás a saúde da mulher era restrita apenas a complicações gravídicas e maternas. As lutas feministas apontaram passagem para a construção de uma Política Nacional de Atenção Integral à Saúde da Mulher no SUS em 2004. Essa política deu abertura para se trabalhar aspectos de humanização do ser mulher e seu bem-estar biopsicossocial (MINISTÉRIO DA SAÚDE, 2004).

Nesse ínterim, pode-se definir hoje a saúde da mulher como sendo o estado completo de bem-estar físico, psíquico, social, espiritual e ambiental, ampliando o modo de ser mulher para além das nuanças concernes à reprodução, sexualidade e maternidade, inserindo este indivíduo nas relações com o meio ambiente, alimentação, moradia, trabalho, renda, prazer e lazer (BRETON DL, 2017).

Levando-se em consideração o rol de aspectos apresentados no tocante a alteridade do ser e pertencer ao engodo do sexo feminino, é importante compreender o conceito de alteridade feminina como sendo a inserção interpessoal da mulher no meio social como indivíduo capaz de resignar sua própria história restituindo seu valor, de forma singular e autônoma, bem como delimitando e vivendo os seus espaço de falar e existir, afirmando, dessa forma, sua feminilidade e rompendo com uma posição secundária em termos de cuidados e de todas as particularidades que respaldam a significância da sua saúde e sobrevivência (COELHO S e PORTO YF, 2013).

\section{Ser mulher na sociedade pós-contemporânea}

Nos últimos anos, a luta pela valorização do ser mulher vem crescendo consideravelmente. Mas, apesar das inúmeras batalhas vencidas, ainda existem obstáculos que dificultam o alcance da liberdade feminina, da conquista de um espaço igualitário, do respeito e dos direitos iguais para todos, os quais infelizmente são dificultados por uma sociedade culturalmente machista (HESSEL BRCBA e FURTADO IMCG, 2019).

A busca da mulher pelo reconhecimento do seu papel na sociedade iniciou-se a muito tempo, um exemplo disso é que por meados de 1970, anos após a segunda guerra mundial, elas começaram a 
adentrar no mercado de trabalho, que até então era completamente formado por homens. Naquela época as mulheres eram vistas apenas como "mulheres do lar" ou "donas de casa", assim, eram ensinadas a zelarem pelo marido, casa e família, sendo mal vistas aquelas que decidiam se aventurar por outros caminhos (SANTOS LG, 2019).

Após o primeiro movimento contrário ao que era proposto na época, as mulheres passaram a lutar diariamente por tudo que acreditavam ter direito, e que até então era negado a elas, luta essa que persiste até os dias atuais, pois apesar de anos reivindicando por uma sociedade mais justa, isso ainda não aconteceu, e em pleno século XXI as mulheres continuam sendo violentadas de diversas formas, o que acarreta na desestabilização do bem-estar biopsicossocial desses seres (LIMA RR, 2020).

Ainda hoje, mulheres continuam sendo oprimidas de várias formas, tendo sua saúde comprometida. Dentre os fatores que contribuem para o adoecimento dessas mulheres destacam-se as violências e a busca pelo corpo perfeito, sendo este último bastante influenciado pela mídia. Dessa forma, todas essas imposições geram angústia àquelas que não atendem ao ideal cultural de ser mulher, fator que mais uma vez acarreta sofrimento psíquico a este grupo (HESSEL BRCBA e FURTADO IMCG, 2019).

Não obstante, a mulher tem buscado sua independência nas várias esferas sociais. Percebe-se uma reconstrução das noções culturais de beleza, envolvendo um desmistificar de padrões de gênero préestabelecidos e evidenciando a compreensão de si própria enquanto um ser político. Em suma, compreende-se que a luta é contínua, objetivando a desestruturação do patriarcado enquanto sistema de opressão. Portanto, vê-se que ser mulher na sociedade pós-contemporânea é desafiador, pois, após a configuração de tantas lutas por respeito e pelo cuidado integral em todos os âmbitos da sua existência, a mulher permanece em busca de equidade na saúde e na alteridade do devir (LIMA RR, 2020).

\section{A atuação do profissional de saúde frente à saúde da mulher}

O Programa de Assistência Integral à Saúde da Mulher (PAISM) do Sistema Único de Saúde (SUS) garante que os cuidados para com este ser sejam humanizados, pautados em princípios e direitos da saúde por completa, equidade de gênero, valorização e apropriação de seu corpo e do seu eu mulher, através de uma assistência qualificada e com equipes multiprofissionais que atuem na vertente da interdisciplinaridade (MACHADO CV, et al., 2017; CONSELHO FEDERAL DE PSICOLOGIA, 2013).

Dessa forma, o enfermeiro, enquanto membro da equipe multiprofissional, deve prestar uma assistência pautada no acolhimento, incluindo consultas individuais, ginecológicas, planejamento familiar, acompanhamento no desenvolvimento gestacional, parturição e puerpério, ações educativas almejando diversidades de temas como sexualidade e estratégias de bem-estar físico-mental-social (CONSELHO FEDERAL DE PSICOLOGIA, 2013). Para alcançar um cuidado humanizado e adequado, este profissional precisa ofertar um atendimento voltado à promoção, prevenção e recuperação da saúde considerando todo ciclo de vida da mulher (VASCONCELOS MFF, et al., 2017; FIGUEIROA M, et al., 2017).

Outro profissional importante para esse processo de evolução e mutação do ser mulher é o psicólogo, tendo em vista que a Psicologia, sobretudo nos estudos de cunho social, encontra-se inscrita num campo de problematizações no que diz respeito à manutenção de um status quo de questões de gênero, por exemplo, no que tange noções estereotipadas da mulher exclusivamente enquanto mãe-esposa-heterossexual, bem como no desenvolvimento de novas práticas e saberes no SUS (COPETTI AVS e QUIROGA CV, 2018).

Além disso, os profissionais precisam conhecer o ser mulher e suas alteridades, para que assim ofertem uma assistência direcionada que comtemple suas necessidades. Sabe-se que esse ser passa por várias mudanças ao longo da vida, desde a adolescência até a senescência. Todas essas mudanças podem gerar medo e insegurança, tornando a vivência mais dolorosa que o necessário (HEYMANN J, et al., 2019).

Algumas mulheres apresentam resistência diante de cuidados relacionados a sexualidade e reprodução, acredita-se que isso aconteça devido aos tabus sociais que envolvem estes temas. Portanto, enfatiza a necessidade do planejamento de ações em saúde que atuem na tentativa de rever esses preconceitos. É importante que os profissionais da saúde, principalmente enfermeiros e psicólogos, iniciem o acompanhamento das mulheres o quanto antes, objetivando educa-las sobre as nuances do ser mulher $\mathrm{e}$ facilitar a autonomia sobre o seu corpo e o seu ser (FIGUEIROA M, et al., 2017). 
Assim, ressalta-se que é função desses profissionais inserir a mulher nesse contexto de alteridade e sexualidade, abordando uma comunicação e ausculta ativa, auxiliando em dúvidas sobre sexo, corpo e desmistificando falsas informações, respeitando as crenças e culturas (FERNANDES L, et al., 2016). Portanto, é sobre produzir saúde juntamente com as mulheres, configurando uma rede intensa de fazeres, saberes e afetos, expandindo campos existenciais de cada modo de ser dentro das diversidades de cada sujeito (PATTERSON LE, 2019).

\section{CONSIDERAÇÕES FINAIS}

O presente estudo tornou evidente o papel do ser mulher na contemporaneidade e como esta vem vivendo a sua alteridade, bem como, destacou as lutas vivenciadas por essa classe na busca de espaço, respeito e igualdade no tocante aos cuidados biopsicossociais, tendo como base a política de saúde que a atende. Tem-se percebido a atuação pertinente e o empenho da Enfermagem e da Psicologia frente à saúde da mulher, apesar dos desafios enfrentados por ambos os campos, atitude esta que torna visível o quanto é singular o cuidado multiprofissional concernente ao cuidado com corpo, a partir de uma visão holística. Diante do exposto, ressalta-se que apesar de todas as batalhas, as mulheres vivem em uma busca constante por respeito em todas as esferas sociais, sejam elas, no lar, no trabalho, na saúde, no seu dia-a-dia. Diante do exposto, percebeu-se que ser mulher no aqui e agora é sempre uma busca ativa, um vir-a-ser de incompletudes que define um espaço de fala de toda uma coletividade em existência oprimida.

\section{REFERÊNCIAS}

1. BEAUVOIR S. O segundo sexo: experiência vivida. São Paulo: Difusão Europeia do Livro, 1967.

2. BENHABIB SDC. Feminismo como crítica da modernidade. Trad.nathanael da costa caixeiro. Rio de janeiro: editora rosa dos tempos, 1987.

3. BOTTI NCL, et al. Condições de saúde de mulheres com transtorno mental. Rev. Da rede de enfermagem do nordeste, 2013; 14(6): 209-1216.

4. CONSELHO FEDERAL DE PSICOLOGIA (CFP). Documento ํํํㅜㅗ Brasília, DF de 2013. Documento de Referência Para Atuação de Psicólogas (os) em Serviços de Atenção À Mulher em Situação de Violência. Brasília, Brasil, 2013. Disponível em: http://www.crpsp.org.br/portal/comunicacao/artes-graficas/arquivos/2013-CREPOP-ViolenciaMulher.pdf. Acesso em: 03 de julho de 2020.

5. CONSELHO NACIONAL DE SECRETARIAS MUNICIPAIS DE SAÚDE (CONASEMS). Saúde da mulher a construção do cuidado integral e a desconstrução do machismo. Brasília, Brasil, 2019. Disponível em: http://conselho.saude.gov.br/ultimas-noticias-cns/215-saude-da-mulher-a-construcao-do-cuidado-integral-e-adesconstrucao-do-machismo. Acesso em: 01 de julho de 2020.

6. BRETON DL. Antropologia do corpo e modernidade. Petrópolis: Vozes, 2017.

7. BUTLER, J. Problemas de gênero: feminismo e subversão da identidade. Tradução de Renato Aguiar. Rio de janeiro: Civilização. Brasileira, 2003.

8. CHACON DRA. Rosto e responsabilidade na filosofia da alteridade em Emmanuel Levinas, Porto Alegre. Intuitio, 2015; 8(2): 15-24.

9. COELHO S, PORTO YF. Saúde da mulher. Belo Horizonte: $2^{\circ}$ ed, $2012 ; 115 \mathrm{p}$.

10. COPETTI AVS, QUIROGA CV. A influência da mídia nos transtornos alimentares e na autoimagem em adolescentes. Revista de Psicologia da IMED, 2018; 10(2): 161-177.

11. FERNANDES L, et al. Atuação do Enfermeiro no Gerenciamento do Programa de Assistência Integral à Saúde da Mulher. Rev. Brasileira de Ciências da Saúde, 2016; 20 (3):219-226.

12. FIGUEIROA $M$, et al. A formação relacionada com a sexualidade humana na percepção de estudantes de enfermagem. Rev. Research, 2017; 4(15): 21-30.

13. FRANCISCO AM. A mulher "como o outro": gênero, violência, ética e alteridade. 2013. Dissertação (Mestrado em filosofia). Universidade de Caxias do Sul, Caxias do Sul, 2013; 74 p.

14. HESSEL BRCB, FURTADO IMCG. A influência do padrão de magreza para a mulher na contemporaneidade. Revista psicologia, diversidade e saúde, 2019; 8(1): 75-85.

15. HEYMANN J, et al. Improving health with programmatic, legal, and policy approaches to reduce gender inequality and change restrictive gender norms. Series, 2019; 3(393): 522-3.

16. INSTITUTO NACIONAL DE CANCÊR (INCA). Ministério da saúde (MS). Causas E prevenção. Brasília, Brasil, 2020. Disponível em: https://www.inca.gov.br/. Acesso em: 03 de julho de 2020. 
17. LIMA RR. Segurança pública e violência contra a mulher: uma revisão narrativa. Revista Eletrônica Acervo Científico, 2020; 10: e2995.

18. MACEDO JG, ARRAES R. Autonomia da gestante na escolha de parto na realidade da prestação de assistência médico-hospitalar brasileira. VII jornada de sociologia da saúde, 2013.

19. MACHADO CV, et al. Políticas de saúde no Brasil em tempos contraditórios: caminhos e tropeços na construção de um Sistema universal. Cad. Saúde Pública, 2017; sup 2 (e00129616): 55-80.

20. MARTINO LMS, MARQUES ACS. A comunicação como ética da alteridade: pensando o conceito de Lévinas. São Paulo. Intercom - RBCC, 2019; 42(3): 21-40.

21. MENDES VR, et al. A saúde da mulher no contexto dos direitos sexuais e reprodutivos discutidos em periódicos nacionais. Rev. renome, 2015; 4(2): 61-72.

22. MINISTÉRIO DA SAÚDE (MS). Secretaria de atenção à saúde. Departamento de ações programáticas estratégicas. Política nacional de atenção integral à saúde da mulher: princípios e diretrizes. Brasília, Brasil, 2004. Disponível em: https://conselho.saude.gov.br/ultimas_noticias/2007/politica_mulher.pdf. Acesso em: 01 de julho de 2020.

23. MINISTÉRIO DA SAÚDE (MS). Gabinete do ministro. Rede cegonha. Brasília, Brasil, 2011a. Disponível em https://bvsms.saude.gov.br/bvs/saudelegis/gm/2011/prt1459_24_06_2011.html: Acesso em: 01 de julho de 2020.

24. MINISTÉRIO DA SAÚDE (MS). Gabinete do ministro. Política nacional de atenção integral à saúde da mulher. Brasília, Brasil, 2011b. Disponível em: https://bvsms.saude.gov.br/bvs/publicacoes/politica_nacional_mulher_principios_diretrizes.pdf. Acesso em: 01 de julho de 2020.

25. MOROSINI MVGC, et al. Política nacional de atenção básica 2017: retrocessos e riscos para o sistema único de saúde. Saúde debate, 2018; 42(116): 11-24.

26. SANTOS LG. As relações de trabalho no capitalismo contemporâneo: a inserção da mulher negra no mercado. Caderno humanidades em perspectivas, 2019; 7(3): 112-121.

27. PATTERSON LE; EISENBERG S. O processo de aconselhamento. São Paulo: Martins Fontes, 2019.

28. SALES OP, et al. O sistema único de saúde: desafios, avanços e debates em 30 anos de história. Revista humanidade e inovação, 2019; 6(17): 54-65.

29. SODRÉ MP, et al. Ações de extensão para a promoção do parto humanizado: capacitando agentes comunitários de saúde. Rev. Ciência em extensão, 2017; 13(4): 152-161.

30. VASCONCELOS MFF, et al. Saúde da mulher: o que é poderia ser diferente?. Rev. psicol. polít., 2017; 17(39): 327339. 